\title{
ISOMORPHISM BETWEEN ENDOMORPHISM RINGS OF MODULES OVER A SEMI SIMPLE RING
}

\author{
Hery Susanto $^{1}$, Santi Irawati ${ }^{1}$, Indriati Nurul Hidayah ${ }^{1}$, And \\ IRAWATI $^{2}$ \\ ${ }^{1}$ Mathematics Department, Universitas Negeri Malang, Jalan Semarang 5, \\ Malang 65145, Indonesia \\ hery.susanto.fmipa@um.ac.id, santi.irawati.fmipa@um.ac.id, \\ indriati.nurul.fmipa@um.ac.id \\ ${ }^{2}$ Mathematics Department, Institut Teknologi Bandung, Jalan Ganesha 10, \\ Bandung 40132, Indonesia \\ irawati@math.itb.ac.id
}

\begin{abstract}
Our question is what ring $R$ which all modules over $R$ are determined, up to isomorphism, by their endomorphism rings? Examples of this ring are division ring and simple Artinian ring. Any semi simple ring does not satisfy this property. We construct a semi simple ring $R$ but $R$ is not a simple Artinian ring which all modules over $R$ are determined, up to isomorphism, by their endomorphism rings.

Key words and Phrases: Modules, division ring, simple Artinian ring, semi simple ring
\end{abstract}

\section{Introduction}

Let $M, N$ be modules over a ring $R$ and set of all $R$-homomorphisms from $M$ to $N$ is written $\operatorname{Hom}_{R}(M, N)$. Then $\operatorname{Hom}_{R}(M, N)$ is Abelian group over addition of mapping. Moreover, $\operatorname{End}_{R}(M)=\operatorname{Hom}_{R}(M, M)$ is a ring over addition and composition of mapping, and called endomorphism ring of $M$. In general if two modules are isomorphic then their endomorphism rings are isomorphic. The converse is not true. The Baer-Kaplansky theorem states that two Abelian torsion groups are isomorphic if and only if their endomorphism rings are isomorphic (see $[2]$ and [4]). Ivanov got same result with the Baer-Kaplansky theorem. Let $R$ be the upper triangular ring over a division ring and $\mathcal{F}$ be the category of $R$-modules which have a summand isomorphic to $R$. Then modules in $\mathcal{F}$ are determined, up isomorphism, by their endomorphism rings (see [3]). Our question is what ring

2020 Mathematics Subject Classification: 16D10

Received: 01-11-2018, accepted: 01-04-2019. 
which modules in $R$-MOD are determined, up to isomorphism, by their endomorphism rings? Examples of this ring are division ring and simple Artinian ring. Any semi simple ring does not satisfy this property. We construct a semi simple ring $R$ but $R$ is not a simple Artinian ring which modules in $R$-MOD are determined, up to isomorphism, by their endomorphism rings.

In this paper a ring will be a ring with unity and all modules will be nonzero unital right module except if in special case. Definition of ring, module, and others which are used in this paper refer to [1].

\section{IP-ISOMORPHISM}

In Baer-Kaplansky theorem and Ivanov result, isomorphism between endomorphism rings of two modules always "preserve indecomposable direct summand", which called an IP-isomorphism. For a class of modules which have a decomposition $M=\oplus_{i \in \mathcal{I}} M_{i}$ with property that every indecomposable direct summand of $M$ is contained in the sum of finite number of the $M_{i}$ (we say that $M$ has the finite embedding property), an IP-isomorphism between endomorphism rings of modules give an isomorphism between the modules.

An element $e$ in a ring $R$ is called idempotent if $e^{2}=e$. Two idempotents $e_{1}$ and $e_{2}$ in $R$ are called orthogonal if $e_{1} e_{2}=0=e_{2} e_{1}$. An idempotent $e \neq 0$ in $R$ is called primitive idempotent if $e$ can not be a sum of two nonzero orthogonal idempotent.

Let $M$ be a module over a ring $R$ and $e$ an idempotent in $\operatorname{End}_{R}(M)$. Then $1-e$ is also idempotent in $\operatorname{End}_{R}(M)$. Moreover, $e$ and $1-e$ orthogonal and $M$ has decomposition $M=e M \oplus(1-e) M$. Then the direct summand $e M$ is indecomposable if and only if $e$ is a primitive idempotent in $\operatorname{End}_{R}(M)$ ([1], Lemma 5.11).

Let given $M$ and $N$ be modules over a ring $R$, and $\varphi: M \longrightarrow N$ any $R$ isomorphism. The mapping $\alpha$ which defined

$$
\alpha: \operatorname{End}_{R}(M) \longrightarrow \operatorname{End}_{R}(N), \alpha(\psi)=\varphi \psi \varphi^{-1}
$$

is a ring isomorphism. Furthermore, for any idempotent $e$ in $\operatorname{End}_{R}(M)$ satisfies

$$
\alpha(e) N=\varphi e \varphi^{-1} N=(\varphi e)\left(\varphi^{-1} N\right)=(\varphi e) M=\varphi(e M) .
$$

Therefore

$$
\left.\varphi\right|_{e M}: e M \longrightarrow \alpha(e) N
$$

is a $R$-isomorphism. So $e M \cong \alpha(e) N$, for all $e$ idempotent in $\operatorname{End}_{R}(M)$.

In general, if $\beta: \operatorname{End}_{R}(M) \longrightarrow \operatorname{End}_{R}(N)$ is any ring isomorphism and $e$ is any primitive idempotent in $\operatorname{End}_{R}(M)$ then $\beta(e)$ is also a primitive idempotent in $\operatorname{End}_{R}(N)$ but it must not $e M \cong \beta(e) N$.

Let $M$ and $N$ be modules over a ring $R$. A ring isomorphism

$$
\phi: \operatorname{End}_{R}(M) \longrightarrow \operatorname{End}_{R}(N)
$$


is called $I P$-isomorphism if $\phi(e) N \cong e M$, for all primitive idempotents $e$ in $\operatorname{End}_{R}(M)$. The following proposition gives property that an IP-isomorphism between endomorphism rings will give module isomorphism.

Proposition 2.1. Let $M$ and $N$ be modules over a ring $R$ where $M$ has the finite imbedding property with respect to a decomposition into indecomposable direct summands and $N$ be generated by indecomposable direct summands. Then $M$ and $N$ are isomorphic if and only if there is an IP-isomorphism between $\operatorname{End}_{R}(M)$ and $\operatorname{End}_{R}(N)$.

Proof. See [3] Proposition 1.

\section{Main Results}

Let $\mathbb{Z}_{p}$ and $\mathbb{Z}_{q}$ be fields of integer number modulo $p$ and $q$, respectively, where $p$ and $q$ are different prime numbers. Let

$$
R=\left[\begin{array}{ccc}
\mathbb{Z}_{p} & 0 & 0 \\
0 & \mathbb{Z}_{q} & \mathbb{Z}_{q} \\
0 & \mathbb{Z}_{q} & \mathbb{Z}_{q}
\end{array}\right]=\left\{\left[\begin{array}{ccc}
a & 0 & 0 \\
0 & b & c \\
0 & d & e
\end{array}\right] \mid a \in \mathbb{Z}_{p}, \quad b, c, d, e \in \mathbb{Z}_{q}\right\}
$$

Then $R$ is a semi simple ring $R$ but $R$ is not a simple Artinian ring. As a left modul over itself, $R$ has decomposition over simples modules as follows.

$$
R \cong\left[\begin{array}{c}
\mathbb{Z}_{p} \\
0 \\
0
\end{array}\right] \oplus\left[\begin{array}{c}
0 \\
\mathbb{Z}_{q} \\
\mathbb{Z}_{q}
\end{array}\right] \oplus\left[\begin{array}{c}
0 \\
\mathbb{Z}_{q} \\
\mathbb{Z}_{q}
\end{array}\right]=\left[\begin{array}{c}
\mathbb{Z}_{p} \\
0 \\
0
\end{array}\right] \oplus\left[\begin{array}{c}
0 \\
\mathbb{Z}_{q} \\
\mathbb{Z}_{q}
\end{array}\right]^{(2)}
$$

Proposition 3.1. Let $M$ and $N$ be modules over the ring $R$ in (1). Then every ring isomorphism between $\operatorname{End}_{R}(M)$ and $\operatorname{End}_{R}(N)$ is an IP-isomorphism.

Proof. Let $\phi: \operatorname{End}_{R}(M) \rightarrow \operatorname{End}_{R}(N)$ be any ring isomorphism and $e$ be any primitive idempotent in $\operatorname{End}_{R}(M)$. We will be shown that $e M \cong \phi(e) N$. Because $e$ primitive idempotent in $\operatorname{End}_{R}(M)$ then

$$
e M \cong\left[\begin{array}{c}
\mathbb{Z}_{p} \\
0 \\
0
\end{array}\right] \text { or } e M \cong\left[\begin{array}{c}
0 \\
\mathbb{Z}_{q} \\
\mathbb{Z}_{q}
\end{array}\right]
$$

and $\phi(e)$ is idempotent primitive in $\operatorname{End}_{R}(N)$. Suppose that $e M \cong\left[\begin{array}{c}\mathbb{Z}_{p} \\ 0 \\ 0\end{array}\right]$ then

$$
\mathbb{Z}_{p} \cong \operatorname{End}_{R}(e M) \cong e \operatorname{End}_{R}(M) e \cong \phi(e) \operatorname{End}_{R}(N) \phi(e) \cong \operatorname{End}_{R}(\phi(e) N) .
$$


Suppose that $\phi(e) N ¥\left[\begin{array}{c}\mathbb{Z}_{p} \\ 0 \\ 0\end{array}\right]$ then $\phi(e) N \cong\left[\begin{array}{c}0 \\ \mathbb{Z}_{q} \\ \mathbb{Z}_{q}\end{array}\right]$. So that $\operatorname{End}_{R}(\phi(e) N) \cong$ $\mathbb{Z}_{q} ¥ \mathbb{Z}_{p}$. So $\phi(e) N \cong\left[\begin{array}{c}\mathbb{Z}_{p} \\ 0 \\ 0\end{array}\right] \cong e M$. In the case of $e M \cong\left[\begin{array}{c}0 \\ \mathbb{Z}_{q} \\ \mathbb{Z}_{q}\end{array}\right]$, with proof as before, we have the same results. So $\phi$ is IP-isomorphism.

Corollary 3.2. Let $M$ and $N$ be modules over the ring $R$ in (1). Then $M \cong N$ if and only if $\operatorname{End}_{R}(M) \cong \operatorname{End}_{R}(N)$.

Proof. Because ring isomorphism between $\operatorname{End}_{R}(M)$ and $\operatorname{End}_{R}(N)$ always in the form of IP-isomorphism and decomposition of semi simple modules fulfills the insertion properties so that according to the Proposition 2.1 obtained $M \cong N$. tained.

As a generalization of the results above, the following propositions are ob-

Proposition 3.3. Let $R$ be a semi simple ring and has decomposition

$$
R \cong I_{1}^{\left(n_{1}\right)} \oplus \cdots \oplus I_{s}^{\left(n_{s}\right)}
$$

where $I_{k}$ is a simple ideal, $I_{k} \cong I_{l}$ if and only if $k=l$, and

$$
R \cong \mathbb{M}_{n_{1}}\left(D_{1}\right) \oplus \cdots \oplus \mathbb{M}_{n_{s}}\left(D_{s}\right)
$$

where $D_{k}$ is a division ring. Then the following properties are equivalent.

(a) Every ring isomorphism $\phi: \operatorname{End}_{R}(M) \longrightarrow \operatorname{End}_{R}(N)$, where $M$ and $N$ are modules over $R$, is a IP-isomorphism.

(b) $\operatorname{End}_{R}\left(I_{k}\right) \supsetneqq \operatorname{End}_{R}\left(I_{l}\right), k \neq l$.

(c) $D_{k} \supsetneqq D_{l}, k \neq l$.

Corollary 3.4. Let $R$ be a semi simple ring which has decomposition (3) and $\operatorname{End}_{R}\left(I_{k}\right) ¥ \operatorname{End}_{R}\left(I_{l}\right), k \neq l$. Then for $M$ and $N$ modules over $R$, we have $M \cong N$ if and only if $\operatorname{End}_{R}(M) \cong \operatorname{End}_{R}(N)$.

Acknowledgement. This research is supported by Dana PNBP Universitas Negeri Malang 2018 organized by LP2M Universitas Negeri Malang. The authors thank the anonymous referees for their valuable suggestions which let to the improvement of the manuscript.

\section{REFERENCES}

[1] Anderson, F. W. and Fuller, K.R.,Rings and Categories of Modules, New York: SpringerVerlag, 1992.

[2] Fuchs, L., Infinite Abelian Groups Vol. II, New York: Academic Press, 1973. 
[3] Ivanov, G., Generalising the Baer-Kaplansky theorem Journal of Pure and Applied Algebra, 133 (1988), 107-115

[4] Kaplansky, I., Infinite Abelian Groups, Ann Arbor: University of Michigan Press, 1954. 